\title{
Observation of Coexisting Dissipative Solitons in a Mode-Locked Fiber Laser
}

\author{
Chengying Bao, ${ }^{1,2}$ Wonkeun Chang, ${ }^{3}$ Changxi Yang, ${ }^{2}$ Nail Akhmediev, ${ }^{3}$ and Steven T. Cundiff ${ }^{1,4, *}$ \\ ${ }^{1}$ JILA, University of Colorado \& National Institute of Standards and Technology, Boulder, Colorado 80309-0440, USA \\ ${ }^{2}$ State Key Laboratory of Precision Measurement Technology and Instruments, \\ Department of Precision Instruments, Tsinghua University, Beijing 100084, China \\ ${ }^{3}$ Optical Sciences Group, Research School of Physics and Engineering, \\ The Australian National University, Canberra ACT 2601, Australia \\ ${ }^{4}$ Physics Department, University of Michigan, Ann Arbor, Michigan 48109, USA
}

(Received 30 April 2015; published 18 December 2015)

\begin{abstract}
We show, experimentally and numerically, that a mode-locked fiber laser can operate in a regime where two dissipative soliton solutions coexist and the laser will periodically switch between the solutions. The two dissipative solitons differ in their pulse energy and spectrum. The switching can be controlled by an external perturbation and triggered even when switching does not occur spontaneously. Numerical simulations unveil the importance of the double-minima loss spectrum and nonlinear gain to the switching dynamics.
\end{abstract}

DOI: 10.1103/PhysRevLett.115.253903

PACS numbers: $42.65 . \mathrm{Tg}, 42.55 . \mathrm{Wd}$

Solitons are mathematically defined as a localized solution of a partial differential equation describing the evolution of a nonlinear integrable system [1]. The concept of solitons is useful for describing physical phenomena ranging from water waves [2] to optical pulses and beams [3]. However, the requirement of integrability ignores dissipation, which is unavoidable in real physical systems. This difficulty with the strict definition of a soliton led to the concept of a "dissipative soliton," which is a solitonlike entity that exists in the presence of gain and loss [4]. The properties of dissipative solitons have only begun to be explored because the systems where they exist have a richer parameter space than integrable systems.

Optical solitons are the subject of many theoretical and experimental studies because it is possible to carefully tailor the system parameters. In optics, both temporal [5] and spatial [6,7] solitons occur. Mode-locked lasers are an important realization of temporal optical solitons. On one hand, dissipative soliton theory provides important insight into the operation of mode-locked lasers, and can help improve their performance for applications including multiphoton microscopy [8], time-domain spectroscopy [9], and frequency combs [10]. On the other hand mode-locked lasers provide an excellent test bed for soliton dynamics because they provide an essentially infinite propagation distance where the pulse can be sampled once per round trip. For example, polarization locked vector solitons were first observed in a mode-locked fiber laser [11] and exploding solitons were observed first in a mode-locked Ti:sapphire laser [12] and then in a mode-locked fiber laser [13]. The concept of dissipative solitons led to modelocked fiber lasers with unprecedented output power levels [14]. Theoretical studies to determine the stability of modelocked lasers have found that multiple solutions can coexist
[15]. Bistability has also been predicted to occur due to higher order nonlinearities [16].

In this Letter, we report the experimental observation of two dissipative soliton solutions coexisting in a modelocked fiber laser. In certain parameter regimes, the laser spontaneously switches between the two solutions on a time scale of several thousand round trips. The two solutions are distinguished by their pulse energy, center frequency, and spectral width. The switching between the solutions can be controlled by injecting a control signal that modulates the gain by depleting the excited state population. In addition, the control signal can trigger switching in regimes where it does not happen spontaneously. Numerical simulations display similar spontaneous switching dynamics. These results give important new insight into the dynamics of mode-locked lasers that will aid in improving their performance. In addition, the observation that the switching can be triggered has important implications for the response of these lasers to noise, which may be strongly amplified if the laser happens to be in a parameter regime where a small perturbation can trigger it to switch between solutions.

The fiber laser uses erbium doped fiber for gain and is mode locked using nonlinear polarization rotation $[17,18]$. The laser and experimental setup are described in more detail in the Supplemental Material [19] and are similar to the setup used to determine the quantum limits to the comb linewidth for a mode-locked fiber laser [20]. The output power is monitored using a slow photodetector that integrates over the individual pulses and with a monochromator followed by a photodetector. As the pump power is increased, a transition to a stable mode-locked state is observed. In this state there is a pulse circulating in the cavity that is a dissipative soliton solution balancing the linear, nonlinear and dissipative effects in the laser. 


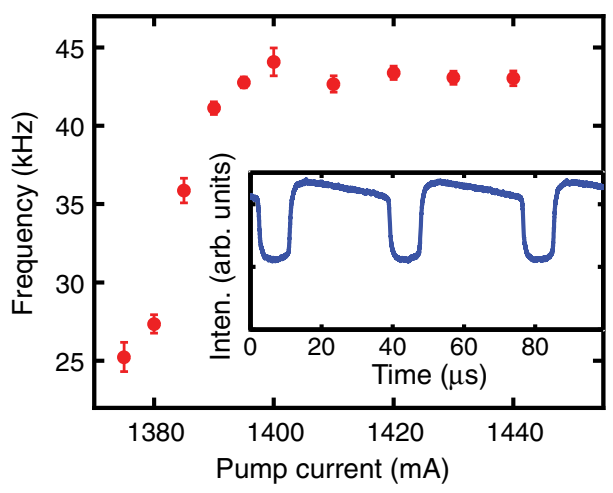

FIG. 1 (color online). Frequency of intensity modulation as a function of pump power. Inset shows output intensity as a function of time for a modulation of $27 \mathrm{kHz}$.

However, a second transition is observed at higher pump power (pump current above $1375 \mathrm{~mA}$ ), above which the output power is periodically modulated, as shown in Fig. 1. Depending on the pump power, the modulation occurs at a frequency ranging from 25 to $45 \mathrm{kHz}$, which corresponds to thousands of round trips as the laser has a $96 \mathrm{MHz}$ repetition rate. The waveform of the intensity consists of abrupt jumps between two power levels, with slow variation at each power level (see inset to Fig. 1).

The optical spectrum exhibits similar bistability. The temporal evolution of the spectrum is reconstructed by filtering out individual spectral slices using a monochromator and recording their dynamics. To synchronize the different spectral slices, the pulse intensity signal is used to trigger the capture of a waveform. The resulting map of the temporal evolution of the spectrum is shown in Fig. 2(a). It

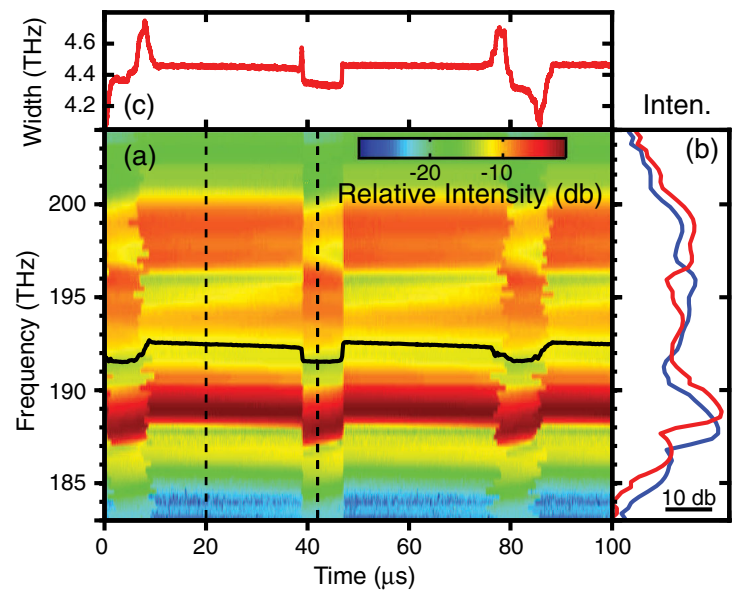

FIG. 2 (color online). Experimentally measured spectral dynamics of the mode-locked fiber laser. (a) Intensity measured as a function of time and frequency. The center frequency (first moment) is plotted as a black line. (b) Two representative spectra corresponding to 20 (red line) and 42 (blue line) $\mu$ s [times indicated by dashed vertical lines in panel (a)]. (c) Spectral width (square root of the second moment) as a function of time. shows two typical spectral shapes with abrupt transitions between them. The spectra at $20 \mu$ s and $42 \mu$ s are shown in Fig. 2(b), which shows a shift in the center frequency. The black line in Fig. 2(a) is the calculated first moment of the spectrum, which clearly shows a shift of approximately $1 \mathrm{THz}$. Furthermore, these two states also have different spectral width (square root of the second moment of the spectrum) as shown in Fig. 2(c), which has an abrupt change of approximately $0.1 \mathrm{THz}$. The fluctuations in the timing of the transitions are due to slight changes in the switching frequency while the map is being acquired.

This fluctuation of the switching frequency can be stabilized by injecting a control signal into the cavity. The control signal is generated by a cw laser that is sinusoidally modulated; thus, it modulates the gain via stimulated emission when it passes through the erbiumdoped fiber. At a pump current of $1375 \mathrm{~mA}$, the switching frequency fluctuates around $20 \mathrm{kHz}$, indicated by the error bars in Fig. 3(a). When modulation frequency of the control signal is scanned, the switching frequency locks to that of the control signal, within the range from $12 \mathrm{kHz}$ to $25 \mathrm{kHz}$, and the fluctuations are suppressed. Locking still occurs when the pump current is increased, although at higher frequency. Furthermore, the switching frequency can be locked to the subharmonic of the modulation frequency of the control frequency. For instance, when injecting the control signal with a modulation frequency of $48 \mathrm{kHz}$, the switching frequency can be locked to $24 \mathrm{kHz}$ [Fig. 3(b)]. Since the switching phenomena is locking to a subharmonic of the control signal, it is a weaker effect, which is apparent in the larger error bars.

In addition to stabilizing the switching frequency, external perturbations can also trigger switching dynamics in regimes where it does not occur spontaneously. This effect is observed at a pump current of $1370 \mathrm{~mA}$, below the threshold of spontaneous switching. Without external perturbation the laser stays stable. When a control signal

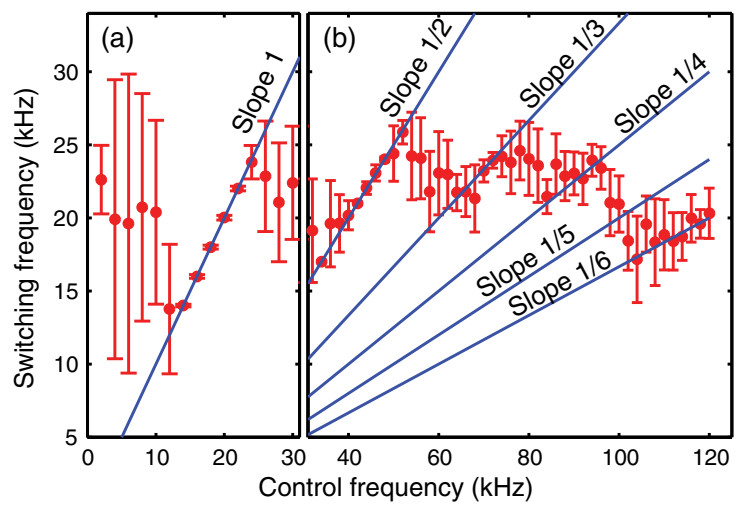

FIG. 3 (color online). Locking of the switching frequency to a control signal. In (a) the locking to the fundamental frequency of the control signal is shown, while subharmonic locking is shown in (b). 


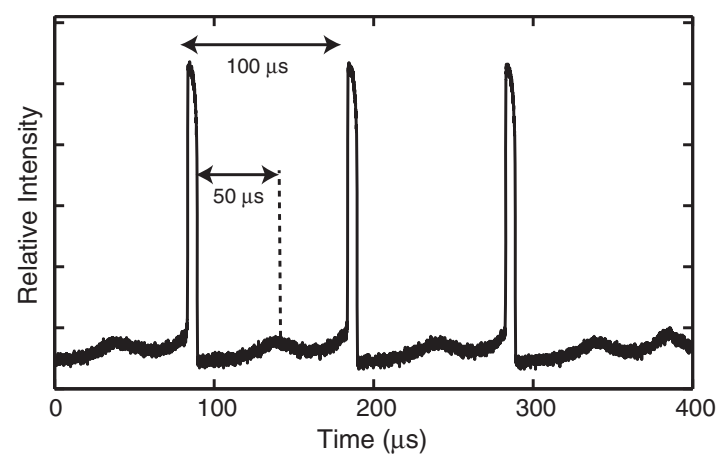

FIG. 4. The response at $1595 \mathrm{~nm}$ to a control signal with a frequency of $20 \mathrm{kHz}$. The pump current is $1370 \mathrm{~mA}$ which corresponds to an absence of spontaneous switching for no control signal.

with intensity modulation at $20 \mathrm{kHz}$ is injected, transients separated by $100 \mu$ s are observed in the output at $1595 \mathrm{~nm}$, as shown in Fig. 4. There is also a weak modulation interleaved with the transients. Although the switching frequency is half the perturbation frequency, this observation shows that switching dynamics can be triggered by external perturbation below the spontaneous threshold. The excitation of the switching dynamics by external perturbation implies that a noise source can be significantly amplified by intracavity pulse dynamics. Such an amplification of noise is detrimental to most applications.

The observations can be interpreted as evidence that multiple stable soliton solutions coexist and the laser is switching between them, either spontaneously or triggered by the control signal. To determine if the observed phenomenon is general and occurs in the theoretical description of the dissipative soliton solutions for a mode-locked laser, we carry out numerical investigation of the pulse dynamics in the fiber laser based on the master equation approach [21]. We use the split step propagation technique that converges to stable solutions. The quartic spectral loss profile has the potential to allow for the switching of the pulse's center frequency between two values. Hence, we use the quintic complex SwiftHohenberg equation (CSHE) as the governing equation, which is given below in its normalized form [22,23].

$$
\begin{aligned}
i \psi_{z} & +\frac{D}{2} \psi_{t t}+\eta|\psi|^{2} \psi+\nu|\psi|^{4} \psi \\
& =i \delta \psi+i \beta \psi_{t t}+i \sigma \psi_{t t t t}+i \epsilon|\psi|^{2} \psi+i \mu|\psi|^{4} \psi,
\end{aligned}
$$

where $\psi$ is the complex envelope of the optical field in the time frame $t$ moving with the pulse, and $z$ is related to the cavity round-trip number. The subscripts denote the derivatives. $D$ is the dispersion parameter where a positive value represents the anomalous dispersion regime and a negative value indicates the normal dispersion regime. $\eta$ and $\nu$ are responsible for the cubic and quintic nonlinearity, respectively. The linear gain or loss spectrum is described by the terms involving $\delta, \beta$ and $\sigma$, while the nonlinear gain or loss is accounted for in the terms containing $\epsilon$ and $\mu$.

Setting $\delta=-0.5, \beta=-0.3$ and $\sigma=-0.05$ introduces a linear loss spectrum that is symmetric around the reference frequency $f_{0}$ with two loss minima at $f-f_{0}= \pm 0.275$, which corresponds to the birefringent filter in the cavity. Note that the loss is not minimum at $f_{0}$. We solved Eq. (1) using an initial condition whose spectrum is centered at $f_{0}$. We tuned the other parameters to $D=-1.0, \eta=1.0$, $\nu=-0.01, \epsilon=0.354$ and $\mu=-0.019$ to obtain a dissipative soliton that pulsates between two frequencies as shown in Fig. 5. The numerical simulation presented here is an illustrative example of the experiment, and the equation parameters are chosen to fit roughly the parameters of the laser. This effect does exist for several sets of parameters of the Swift-Hohenberg equation. In particular, parameter $\nu$ can be chosen to be zero as in previous work [24]. However, it is essential that parameter $\sigma$ is negative, which is a significant extension of the model of Renninger et al. [24]. The unit frequency detuning $f$ and the unit time delay $t$ in our numerical simulations can be determined from the setup, which correspond to $12 \mathrm{THz}$ and $83 \mathrm{fs,} \mathrm{respectively.}$ Hence, the quantitative features of the simulation dynamics are roughly in accordance with the experimentally observed spectral shift of $1 \mathrm{THz}$ and the pulse width of $84 \mathrm{fs}$.

The switching of the soliton's frequency is symmetric around $f_{0}$ for the case shown in Fig. 5(a). The soliton's energy stays predominantly on one side of the spectrum for a certain number of round trips, and then switches to the other side and stays there for the same number of round trips. In the time domain, the frequency switching causes the soliton to be periodically delayed and advanced with respect to the moving time frame as shown in Fig. 5(b).

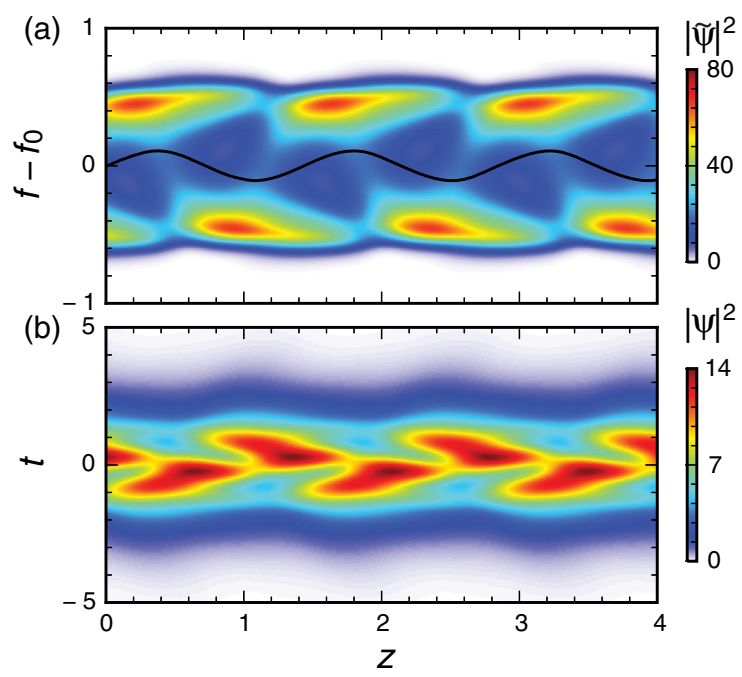

FIG. 5 (color online). (a) Spectral and (b) temporal evolution of the frequency pulsating soliton obtained by solving Eq. (1) with $D=-1.0, \eta=1.0, \nu=-0.01, \delta=-0.5, \beta=-0.3, \sigma=-0.05$, $\epsilon=0.354$, and $\mu=-0.019$. The black solid line in (a) shows the first moment of the spectrum. 


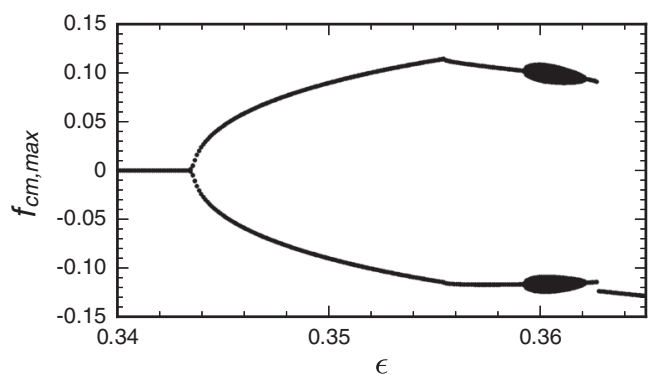

FIG. 6. A bifurcation diagram of the maximum frequency shifts for varying $\epsilon$. The top and bottom branches indicate the maximum shifts toward the blue side and red side, respectively. Other parameters are the same as those in Fig. 5.

We also generate a bifurcation diagram of the maximum frequency shifts as a function of $\epsilon$ (Fig. 6) to study how the frequency switching evolves. A fixed soliton is observed for $\epsilon<0.343$. Past this point, a Hopf bifurcation occurs causing the soliton to shift its frequency periodically between two values that are located symmetrically around $f_{0}$ as the example shown in Fig. 5. When $\epsilon$ reaches 0.356 the symmetry of the bifurcation is broken. The soliton's frequency still shifts between two values on both sides of $f_{0}$, but asymmetrically such that there is a preferred side, the red side for the case shown in Fig. 6. An example of the asymmetric solution from this branch is shown in Fig. 7. The soliton switches farther to the red side than the blue side. In the case of asymmetric switching, it takes the soliton a longer time to switch than the symmetric case.

As $\epsilon$ is increased further, a second frequency appears $0.359<\epsilon<0.362$. The mix of multiple incommensurate frequencies results in a continuous range of values for $f_{\text {cm, max }}$. Such multifrequency dynamics of a dissipative soliton was also observed in a cubic-quintic complex Ginzburg-Landau equation for a creeping soliton forming a zig-zag motion that involves multiple frequencies [25]. However, the primary (higher) frequency component was responsible for the energy pulsation in the former study, whereas it is bound to the switching of the soliton's frequency here. The frequency switching dynamics ceases for $\epsilon>0.3627$ and the soliton returns to its fixed state, but it now finds a new center frequency to stay near the one of the minima in the linear loss spectrum.

The numerical studies show that the linear loss spectrum strongly influences the excitation of switching dynamics. Moreover, since $\epsilon$ is related to nonlinear gain or loss of the laser it depends on the pump power and mode-locking state. Then such a coexistence of dissipative solitons only happens when the laser has certain specific parameters, e.g., a proper loss spectrum, and operates in certain regimes, e.g., certain pump level.

In conclusion, we have observed the coexistence of dissipative soliton solutions in a mode-locked Er-doped fiber laser, characterized by a different pulse energy and center frequency. The center frequency of the soliton

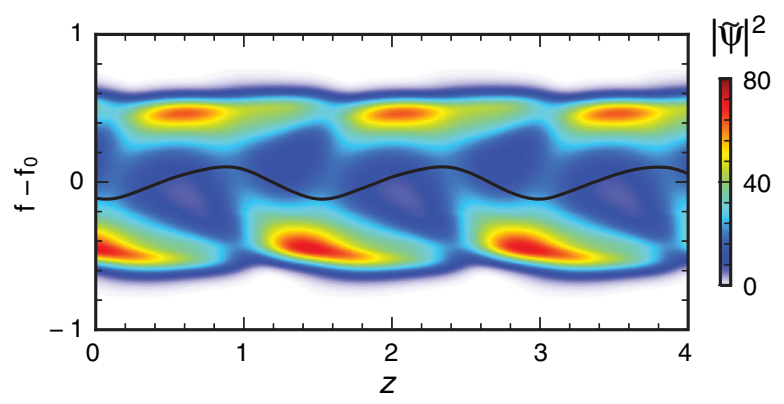

FIG. 7 (color online). Spectral evolution of the asymmetrically frequency pulsating soliton obtained at $\epsilon=0.3585$. The solution has a preferred side towards the red for the case shown here. The black solid line indicates $f_{\mathrm{cm}}$.

periodically switches between two well separated values in thousands of round trips. The frequency of the switching behavior can be locked to an external control signal. Furthermore, the control signal can trigger switching behavior in a regime where it does not happen spontaneously. Numerical simulations exhibit similar switching dynamics. The linear loss spectrum and pulse energy is critical for the presence of the switching. The experimental and theoretical demonstration that dissipative soliton solutions can coexist gives important new insight into dissipative solitons and can help in understanding the noise properties of mode-locked lasers.

C. Bao and C. Yang acknowledge support from National Natural Science Foundation of China (61177046, 61377039). W. Chang and N. Akhmediev acknowledge the support of the Australian Research Council (DE130101432 and DP150102057).

*cundiff@umich.edu

[1] V. F. Zakharov and A. B. Shabat, Sov. Phys. JETP 34, 62 (1972).

[2] J. S. Russell, http://www.macs.hw.ac.uk/ chris/Scott-Russell/ SR44.pdf (1844).

[3] M. Remoissenet, Waves called Solitons: Concepts and Experiments (Springer-Verlag, Berlin-New York, 1999).

[4] Dissipative Soltions edited by N. Akhmediev and A. Ankiewicz (Springer, New York, 2005).

[5] A. Hasegawa and F. Tappert, Appl. Phys. Lett. 23, 142 (1973).

[6] L. A. Lugiato and R. Lefever, Phys. Rev. Lett. 58, 2209 (1987).

[7] J. U. Kang, G. I. Stegeman, J. S. Aitchison, and N. Akhmediev, Phys. Rev. Lett. 76, 3699 (1996).

[8] C. Xu and F. W. Wise, Nat. Photonics 7, 875 (2013).

[9] S. T. Cundiff and S. Mukamel, Phys. Today 66, 44 (2013).

[10] S. T. Cundiff and J. Ye, Rev. Mod. Phys. 75, 325 (2003).

[11] S. T. Cundiff, B. C. Collings, N. N. Akhmediev, J. M. Soto-Crespo, K. Bergman, and W. H. Knox, Phys. Rev. Lett. 82, 3988 (1999). 
[12] S. T. Cundiff, J. M. Soto-Crespo, and N. Akhmediev, Phys. Rev. Lett. 88, 073903 (2002).

[13] A. F. J. Runge, N. G. R. Broderick, and M. Erkintalo, Optica 2, 36 (2015).

[14] K. Kieu, W. H. Renninger, A. Chong, and F. W. Wise, Opt. Lett. 34, 593 (2009).

[15] S. Wang, A. Docherty, B. S. Marks, and C. R. Menyuk, J. Opt. Soc. Am. B 31, 2914 (2014).

[16] A. E. Kaplan, Phys. Rev. Lett. 55, 1291 (1985).

[17] K. Tamura, E. P. Ippen, H. A. Haus, and L. E. Nelson, Opt. Lett. 18, 1080 (1993).

[18] L. Nelson, D. Jones, K. Tamura, H. Haus, and E. Ippen, Appl. Phys. B 65, 277 (1997).
[19] See Supplemental Material at http://link.aps.org/ supplemental/10.1103/PhysRevLett.115.253903 for further experimental details.

[20] C. Bao, A. C. Funk, C. Yang, and S. T. Cundiff, Opt. Lett. 39, 3266 (2014).

[21] H. A. Haus, IEEE J. Quantum Electron. 11, 736 (1975).

[22] J. M. Soto-Crespo and N. Akhmediev, Phys. Rev. E 66, 066610 (2002).

[23] E. Ding and J. N. Kutz, J. Opt. Soc. Am. B 26, 2290 (2009).

[24] W. H. Renninger, A. Chong, and F. W. Wise, Phys. Rev. A 77, 023814 (2008).

[25] W. Chang, A. Ankiewicz, N. Akhmediev, and J. M. Soto-Crespo, Phys. Rev. E 76, 016607 (2007). 